
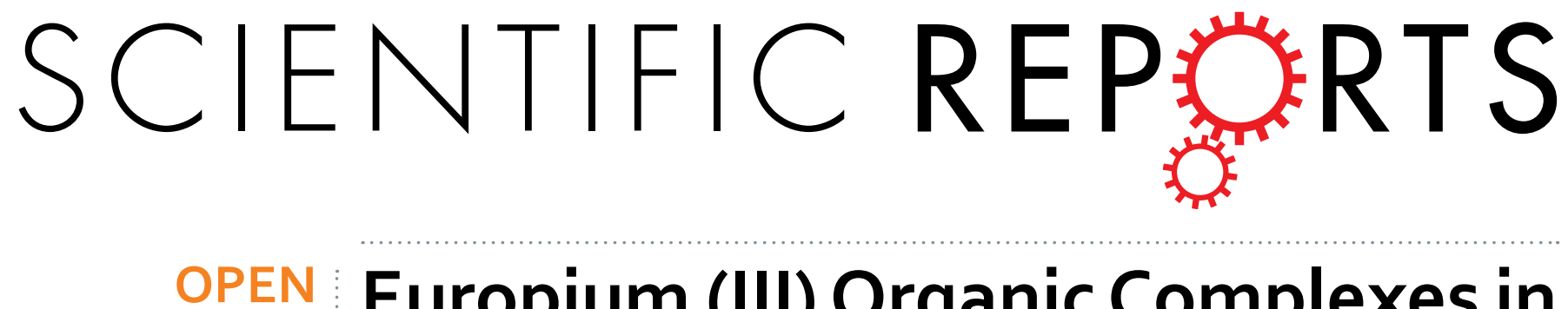

\title{
Europium (III) Organic Complexes in Porous Boron Nitride Microfibers: Efficient Hybrid Luminescent \\ Material
}

Received: 18 July 2016

Accepted: 15 September 2016

Published: 30 September 2016

\author{
Jing $\mathrm{Lin}^{1,2}$, Congcong Feng ${ }^{1,2}$, Xin He ${ }^{1,2}$, Weijia Wang ${ }^{1,2}$, Yi Fang ${ }^{1,2}$, Zhenya Liu ${ }^{1,2}$, Jie $\mathrm{Li}^{1,2}$, \\ Chengchun Tang ${ }^{1,2}$ \& Yang Huang ${ }^{1,2}$
}

We report the design and synthesis of a novel kind of organic-inorganic hybrid material via the incorporation of europium (III) $\beta$-diketonate complexes ( $\mathrm{Eu}(\mathrm{TTA})_{3}, \mathrm{TTA}=2$-thenoyltrifluoroacetone) into one-dimensional (1D) porous boron nitride (BN) microfibers. The developed Eu(TTA) $@$ @BN hybrid composites with typical 1D fibrous morphology exhibit bright visible red-light emission on UV illumination. The confinement of $\mathrm{Eu}(T T A)_{3}$ within pores of $\mathrm{BN}$ microfibers not only decreases the aggregation-caused quenching in solid Eu(TTA $)_{3}$, but also improves their thermal stabilities. Moreover, The strong interactions between $\mathrm{Eu}(\mathrm{TTA})_{3}$ and porous $\mathrm{BN}$ matrix result in an interesting energy transfer process from BN host to TTA ligand and TTA ligand to $\mathrm{Eu}^{3+}$ ions, leading to the remarkable increase of red emission. The synthetic approach should be a very promising strategy which can be easily expanded to other hybrid luminescent materials based on porous BN.

Researches on organic-inorganic hybrid materials have gathered immense attention because of their potential applications in various fields such as sensing, light-emitting, lithium ion battery, etc ${ }^{1-5}$. The combination of organic and inorganic components at a nanometer scale could allow us to generate unique properties in such complex systems. For example, encapsulating organic dyes into porous metal-organic frameworks (MOFs) can efficiently enhance their fluorescence quantum efficiency and tuning the emission color due to the confinement of dyes in the pores of $\mathrm{MOFs}^{2}$.

Trivalent lanthanide complexes with organic ligands are of great interest due to their outstanding luminescent properties. On one hand, lanthanide complexes could possess of characteristic and narrow emission bands with a full width at half maximum of less than $10 \mathrm{~nm}$ and long-lived excited states gaining from the luminescent centres of trivalent lanthanide ions $\left(\mathrm{Ln}^{3+}\right)$. On the other hand, differing from the $\mathrm{Ln}^{3+}$ ions which have poor light absorption abilities due to the forbidden intraconfiguration $f$ - $f$ transition, lanthanide complexes exhibit sensitized emission because the organic ligands have large molar absorption coefficients and can effectively increase light absorption by "antenna effect" 6,7 . In spite of the outstanding luminescent properties of lanthanide complexes, the practical application of these complexes has still been limited due to their poor thermal stability and proccessibility ${ }^{8}$. To solve this problem, introducing lanthanide complexes in a stable porous matrix to form organic-inorganic hybrid materials has been intensively studied. So far, inorganic porous materials, such as zeolites ${ }^{9-11}$, mesoporous silica (SBA-15, MCM-41, etc) ${ }^{12-18}$ have been used as the matrix due to their special porous structure and thermal and chemical stabilities. The confinement of lanthanide complexes within porous matrix can not only improve their stabilities, but also decrease the aggregation-caused quenching in lanthanide complex, which would be beneficial for the effective luminescence ${ }^{19}$.

Porous boron nitride (BN) has attractive properties including low density, high thermal conductivity, superior oxidation resistance and chemical inertness. Particularly, the recently breakthrough on the preparation of porous $\mathrm{BN}$ with high specific surface areas and large pore volumes has gained this porous material much attention ${ }^{20-28}$. We envision that porous BN can be an ideal candidate as the matrix for lanthanide complexes. Notwithstanding

${ }^{1}$ School of Materials Science and Engineering, Hebei University of Technology, Tianjin, 300130, P. R. China. ${ }^{2}$ Hebei Key Laboratory of Boron Nitride Micro and Nano Materials, Hebei University of Technology, Tianjin, 300130, P. R. China. Correspondence and requests for materials should be addressed to J.L. (email: linjing@hebut.edu.cn) orY.H. (email: huangyang@hebut.edu.cn) 


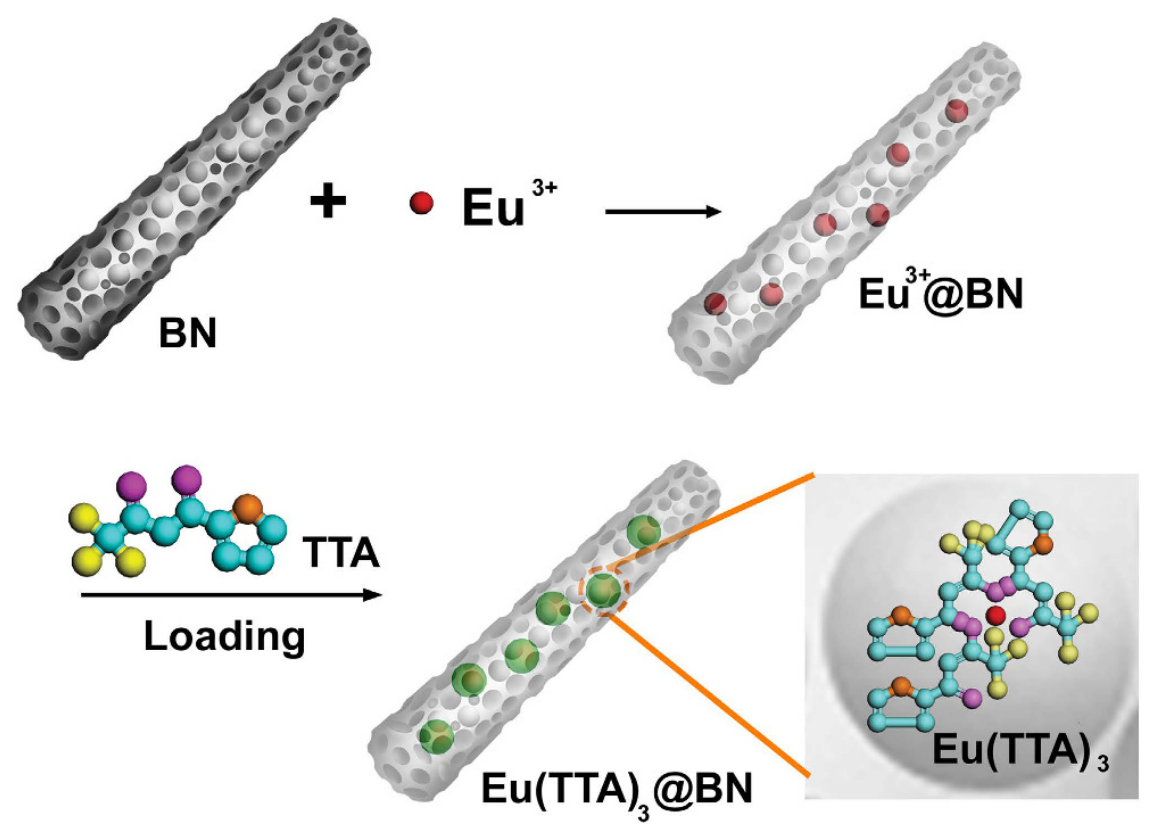

Figure 1. Illustration of the procedure for synthesis of Eu(TTA) ${ }_{3} @ B N$ hybrid microfibers.

the possibility, porous $\mathrm{BN}$ holding characteristic $\mathrm{sp}^{2}$-bonded honeycomb structure is quite different with the traditional oxide porous matrix from both the chemical and physical view of point. So-derived specific interactions with the lanthanide complexes will surely influence the luminescence performance of the constructed hybrid materials. Unfortunately, such lanthanide complex/porous BN hybrid luminescent materials have not been explored so far.

In this paper, we report the design of novel lanthanide complex/porous BN organic-inorganic hybrid materials via the incorporation of europium (III) $\beta$-diketonate complexes (Eu(TTA) $)_{3}, \mathrm{TTA}=2$-thenoyltrifluoroacetone) into porous BN microfibers. The Eu(TTA) ${ }_{3} @ \mathrm{BN}$ composite possesses one-dimensional (1D) fibrous morphology similar with pure porous $\mathrm{BN}$ matrix. The emission intensity and lifetime of $\mathrm{Eu}^{3+}$ ions are significantly enhanced compared with pure europium complexes. The thermal stability of $\mathrm{Eu}(\mathrm{TTA})_{3}$ has also been improved due to the protection of porous $\mathrm{BN}$ matrix. Interestingly, due to the strong interactions between europium complexes and porous $\mathrm{BN}$, efficient energy transfer from BN to TTA ligand and TTA ligand to $\mathrm{Eu}^{3+}$ ions takes place in the hybrid system, leading to the great enhancement of red emission. This novel organic-inorganic hybrid material is envisaged to become highly valuable in lighting devices and biomedical analysis.

\section{Results and Discussion}

Figure 1 illustrates the procedure for synthesis of the organic-inorganic hybrid material Eu(TTA) $3_{3} \mathrm{BN}$. The porous BN products used as the inorganic hosts are mainly some microfibers with main characteristic pore sizes of $\sim 1.3$ and $\sim 3.9 \mathrm{~nm}$. The specific surface area of the porous BN is $\sim 1600 \mathrm{~m}^{2} \mathrm{~g}^{-1}$ and the pore volume is $\sim 0.9 \mathrm{~cm}^{3}$ $\mathrm{g}^{-123}$. The synthesis of Eu(TTA) $)_{3} @ B N$ was realized via a two-step method: the loading of Eu ${ }^{3+}$ ions into porous $\mathrm{BN}$ microfibers was achieved by adsorption of $\mathrm{Eu}^{3+}$ ions in ethanol solution dispersed with porous $\mathrm{BN}$, and then the organic ligands TTA was inserted into $\mathrm{Eu}^{3+} @ \mathrm{BN}$ by a gas diffusion method. Our previous studies indicate that the as-prepared porous $\mathrm{BN}$ exhibits excellent adsorption performance for various metal ions due to their high specific area, large pore volume and dipolar nature of B-N bonds. Especially, the surface of porous BN is negatively charged, so the metal ions can be adsorbed on porous $\mathrm{BN}$ by the electrostatic interactions ${ }^{23,24}$. After obtaining Eu ${ }^{3+} @ B N$ microfibers, the formation of organic-inorganic hybrid Eu(TTA) ${ }_{3} @ B N$ can be realized by coordination reaction between TTA and $\mathrm{Eu}^{3+}$ ions in porous $\mathrm{BN}$ microfibers. X-ray diffraction (XRD) result of hybrid product is quite similar to that of starting porous $\mathrm{BN}$, indicating the amorphous phase of organic $\mathrm{Eu}(\mathrm{TTA})_{3}$ (Supporting Information, Fig. S1).

The microstructures and compositions of the Eu(TTA) $)_{3} @ B N$ hybrid product were further examined in transmission electron microscopy (TEM). A typical low-magnification TEM image (Fig. 2a) indicates that the product keeps fibrous morphology as as-prepared porous BN microfiber. The diameter of the microfiber here is $\sim 0.5 \mu \mathrm{m}$. The bright spots on the microfiber shown in the enlarged TEM image (Fig. 2b) reveal some mesopores with the diameters of tens nm existing in the microfiber. Figure $2 b$ also shows that the surface of the microfiber is quite clear. High resolution TEM image in Fig. $2 c$ shows the edge of the microfiber. The crystallized BN layers (marked by the arrows) is very clear without any adhered substances. High resolution TEM image (Fig. 2d) taking from the centre of the microfibers shows an amorphous phase covered over the microfiber. The amorphous phase could be attributed to the $\mathrm{Eu}(\mathrm{TTA})_{3}$ on BN microfibers. To further confirm our success in introducing Eu(TTA) $)_{3}$, a composition analysis was carried out. Figure $2 \mathrm{e}$, f show scanning transmission electron microscopy (STEM) image and the corresponding energy-dispersive X-ray spectroscopy (EDS) spectrum taken from a single Eu(TTA) ${ }_{3}$ 

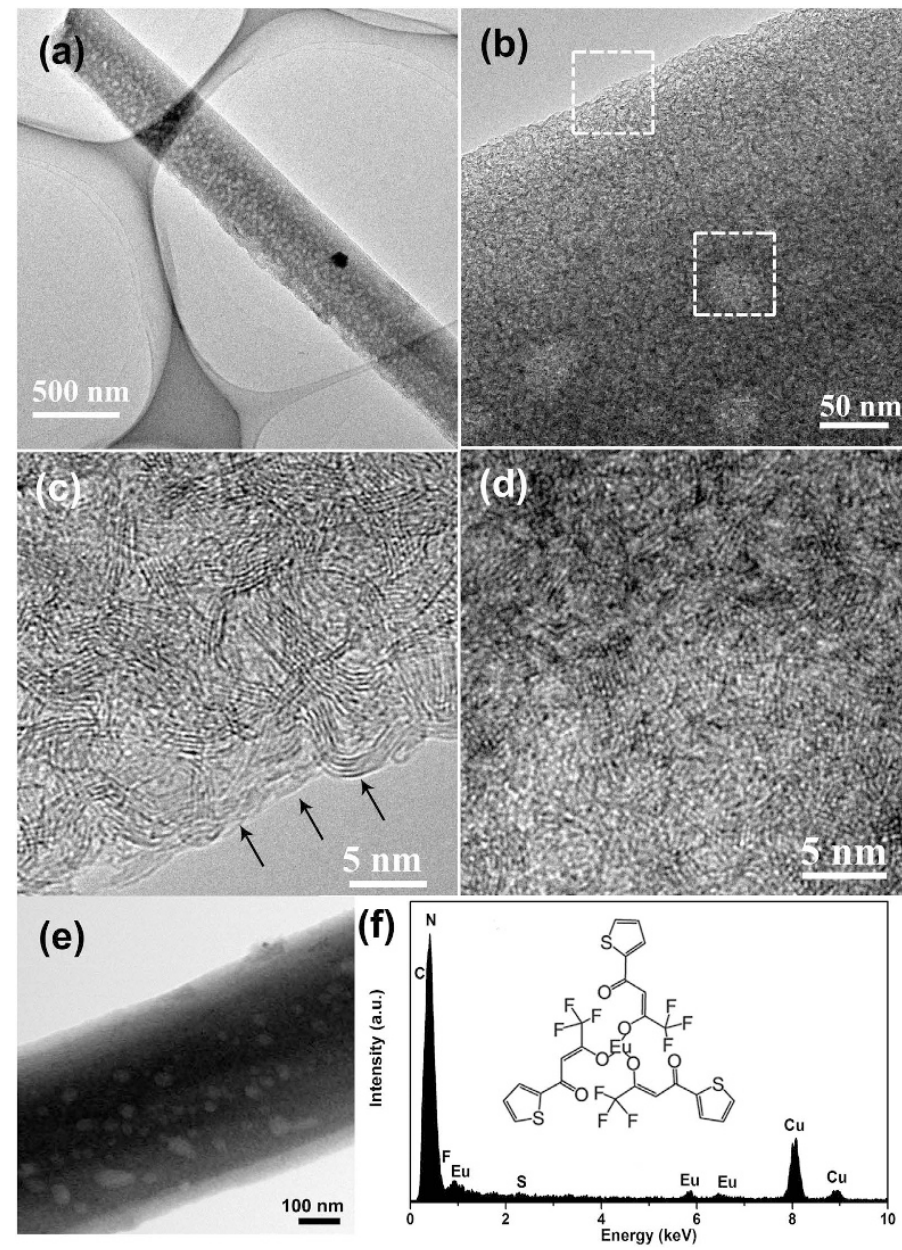

Figure 2. (a) Low-magnification TEM image of Eu(TTA) $@ B N$ hybrid product, revealing the fibrous morphology; (b) Enlarged TEM image of the microfiber; (c, d) HRTEM images taken from the two areas labelled by the two dashed frames marked in (b), respectively; (e) STEM image and (f) the corresponding EDS spectrum taken from a single $\mathrm{Eu}(\mathrm{TTA})_{3} @ \mathrm{BN}$ microfiber.

@BN microfiber. The peaks form C, F, Eu and S elements can be seen clearly. So we can confirm that the organic lanthanide complexes, $\mathrm{Eu}(\mathrm{TTA})_{3}$, have been successfully introduced into the BN microfibers. Besides, the specific surface area of $\mathrm{Eu}(\mathrm{TTA})_{3} @ \mathrm{BN}$ microfibers has been measured as low as $\sim 70 \mathrm{~m}^{2} \mathrm{~g}^{-1}$. Compared with pure porous BN microfibers which possess a very high specific surface area $\left(\sim 1600 \mathrm{~m}^{2} \mathrm{~g}^{-1}\right)$, the significantly decrease of specific surface area implies that the lanthanide complexes has been encapsulated inside the pores of the BN. With a brief summary of the above results, we infer that the lanthanide complexes could be encapsulated inside the pore channels of BN microfiber, rather than coated on their outside surface.

Fourier transform infrared (FTIR) analysis was utilized to study the loading of Eu(TTA) ${ }_{3}$ in porous $\mathrm{BN}$. Figure 3 shows the FTIR spectrum of as-prepared Eu(TTA) @ $_{3} \mathrm{BN}$ sample. The spectra of pure porous BN and $\mathrm{Eu}^{3+} @ \mathrm{BN}$ are also shown for comparison. The FTIR spectrum of porous BN shows peaks at $\sim 1391 \mathrm{~cm}^{-1}$ and $\sim 805 \mathrm{~cm}^{-1}$, corresponding to $\mathrm{B}-\mathrm{N}$ stretching vibrations and $\mathrm{B}-\mathrm{N}-\mathrm{B}$ bending vibrations ${ }^{29}$, respectively. The peak at $\sim 3426 \mathrm{~cm}^{-1}$ is attributed to the vibrations of $\mathrm{B}-\mathrm{OH} / \mathrm{B}-\mathrm{NH}_{2}$ group ${ }^{23}$. In comparison, the FTIR spectrum of Eu $\mathrm{u}^{3+}$ $@ \mathrm{BN}$ is quite similar to that of porous BN, although XPS analysis (Fig. S2, Supporting Information) clearly indicates that the $\mathrm{Eu}^{3+}$ has already been loaded. There is no noticeable shift for all the peaks from porous $\mathrm{BN}$ after incorporating of $\mathrm{Eu}^{3+}$, which means that the $\mathrm{Eu}^{3+}$ ions are adsorbed on $\mathrm{BN}$ by weak interactions, i.e. electrostatic interactions, rather than strong chemical bonding. After loading of $\mathrm{Eu}^{3+}$ and TTA on the porous BN successively, some new absorption peaks at $\sim 1617 \mathrm{~cm}^{-1}, \sim 1093 \mathrm{~cm}^{-1}$ and $\sim 468 \mathrm{~cm}^{-1}$ can be observed. The peak at $\sim 1617 \mathrm{~cm}^{-1}$ is assigned to the $\mathrm{C}=\mathrm{O}$ stretching band which is related to the ligand (TTA); the peak at $\sim 468 \mathrm{~cm}^{-1}$ could be attributed to the stretching $\mathrm{Eu}-\mathrm{O}$ bond, indicating ligands are indeed coordinated with $\mathrm{Eu}^{3+}$ ions through the oxygen in the complex ligands; another peak observed at $\sim 1093 \mathrm{~cm}^{-1}$ is corresponds to the B-N-O group, indicating that the strong interactions also occur between ligand TTA and BN host. The strong interaction between the europium complexes and porous $\mathrm{BN}$ is very import for avoiding the nonhomogeneous distribution and leakage of lanthanide complexes in the porous matrix.

The excitation and emission spectra of $\mathrm{Eu}(\mathrm{TTA})_{3} @ \mathrm{BN}$ microfibers were examined at room temperature. The excitation spectrum monitored at $615 \mathrm{~nm}$ displays a broad band ranging from 250 to $400 \mathrm{~nm}$ (Fig. 4a). The absence of sharp peaks corresponding to the $f-f$ transitions of $\mathrm{Eu}^{3+}$ ions indicates that effective energy transfer 


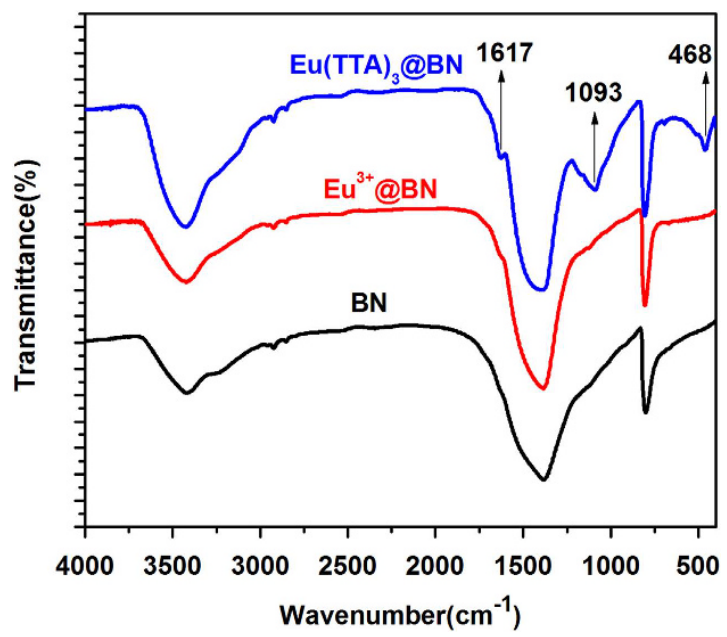

Figure 3. FTIR spectra of porous $\mathrm{BN}(\mathbf{a}), \mathrm{Eu}^{3+} @ \mathrm{BN}(\mathbf{b})$ and $\mathrm{Eu}(\mathrm{TTA})_{3} @ \mathrm{BN}(\mathbf{c})$ samples.
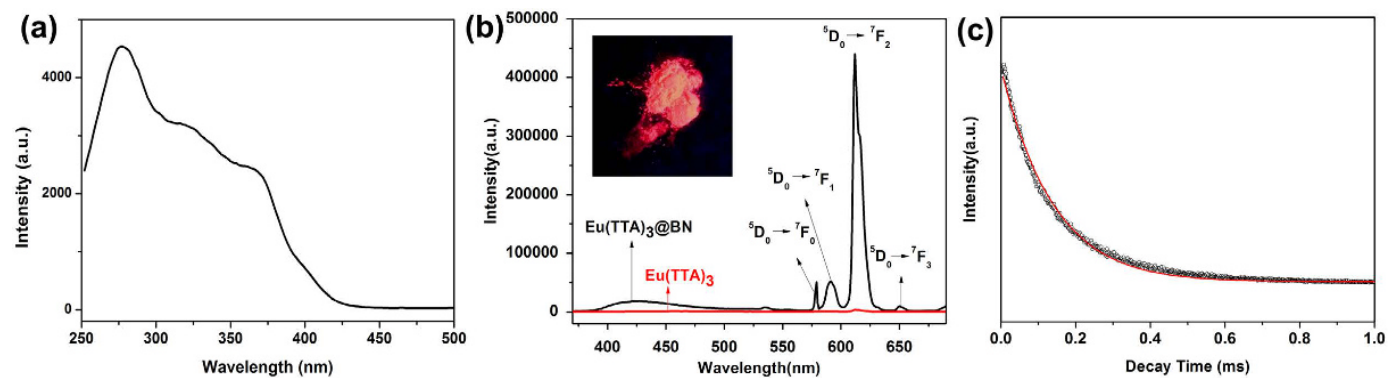

Figure 4. (a) Excitation spectrum of Eu(TTA) $)_{3} @ B N$ samples monitored at $615 \mathrm{~nm}$. (b) Emission spectra of Eu(TTA) $)_{3} @ B N$ and solid Eu(TTA $)_{3} \cdot \mathrm{nH}_{2} \mathrm{O}$ samples excited by $280 \mathrm{~nm}$. (inset) Photo image of Eu(TTA) @ $_{3} \mathrm{BN}$ excited by UV light, showing intense red light emission. (c) Decay curve of Eu(TTA) @ BN sample.

takes place to $\mathrm{Eu}^{3+}$ ions ${ }^{8}$. Upon excited at $280 \mathrm{~nm}$, the Eu(TTA) ${ }_{3} @ \mathrm{BN}$ exhibits intense red emission with characteristic peaks of 581, 591, 615 and $653 \mathrm{~nm}$, which correspond to the excited state ${ }^{5} \mathrm{D}_{0} \rightarrow{ }^{7} \mathrm{~F}_{\mathrm{J}}(\mathrm{J}=0-3)$ of Eu ${ }^{3+}$ ions, respectively (Fig. 4b). To make comparisons, we also measured the emission spectrum of pure $\mathrm{Eu}(\mathrm{TTA})_{3} \cdot \mathrm{nH}_{2} \mathrm{O}$ in the solid state. As shown in Fig. 4b, very weak red emission can be observed in solid Eu(TTA) $)_{3} \cdot \mathrm{nH}_{2} \mathrm{O}$. The results demonstrate that after loading of $\mathrm{Eu}(\mathrm{TTA})_{3}$ in $\mathrm{BN}$ host, the nonradiative energy transfer process between $\mathrm{Eu}(\mathrm{TTA})_{3}$ can be successfully restrained, which would otherwise quench the red emission. The intense red light emission can be easily visible by eye upon $365 \mathrm{~nm}$ UV excitation, as shown in inset of Fig. $4 \mathrm{~b}$. The decay curve of $\mathrm{Eu}(\mathrm{TTA})_{3} @ \mathrm{BN}$ under excitation of $280 \mathrm{~nm}$ is shown in Fig. 4c. The curve can be well fitted mono-exponentially and the value of lifetime $\tau$ is calculated to be $0.361 \mathrm{~ms}$. This value is longer than lifetime of pure $\mathrm{Eu}(\mathrm{TTA})_{3} \cdot \mathrm{nH}_{2} \mathrm{O}$ ( $\tau=0.21 \mathrm{~ms}$, shown in Supporting Information, Fig. S3), which can be attributed to the inhibition of nonradiative deactivation pathways by confinement in the pores of $\mathrm{BN}_{\text {host }}{ }^{8}$.

In order to further clarify the reason for the great enhancement of red emission in the Eu(TTA) $)_{3}$ BN system, we compare the photoluminescence (PL) of pure BN, Eu ${ }^{3+} @ B N$ and Eu(TTA) $3 @ B N$ samples, as shown in Fig. 5a. Pure porous BN microfibers exhibit strong UV emission centered at $\sim 320 \mathrm{~nm}$, which can be attributed to the defect-related centers or intrinsic impurities in $\mathrm{BN}^{30}$. After adsorption of $\mathrm{Eu}^{3+}$, the $\mathrm{Eu} \mathrm{u}^{3+} @ \mathrm{BN}$ sample shows similar UV emission band and a very weak peak at $\sim 613 \mathrm{~nm}$, which corresponds to the red emission of Eu ${ }^{3+}$ ions. Since the $f-f$ transitions of lanthanide ions are spin forbidden, it is hard to generate efficient luminescence emission by direct excitation. Through coordination to TTA ligands, the red emission intensity of Eu(TTA) $)_{3}$ $@ B N$ is significantly enhanced, which can be attributed to the "antenna effect" ${ }^{1,32}$. Effective energy transfer from TTA ligands to $\mathrm{Eu}^{3+}$ ions takes place, resulting in sensitized red emission. Interestingly, we also find the UV emission of BN host disappears in Eu(TTA) $)_{3} @ \mathrm{BN}$, and a weak broad band centered at $450 \mathrm{~nm}$ can be obtained. The $\sim 450 \mathrm{~nm}$ band origins from the $\mathrm{C}$ - and/or O-related impurities in $\mathrm{BN}^{33,34}$. After coordination of TTA ligands with $\mathrm{Eu}^{3+}$ ions in $\mathrm{BN}$ host, the formation of $\mathrm{B}-\mathrm{N}-\mathrm{O}$ bonds makes it possible to obtain the weak blue emission from $\mathrm{BN}$. Besides, the great suppressed UV emission indicates effective energy transfer from BN host to Eu(TTA) may be occurred. As shown in Fig. 5b, the absorption spectrum of TTA shows a broad band between 200 and $450 \mathrm{~nm}$, while the pure BN host exhibits strong UV emission centered at $\sim 320 \mathrm{~nm}$. The spectral overlap between the emission of BN host and absorption of TTA results in an efficient BN-to-TTA energy transfer behavior. PL maps of $\mathrm{BN}$ host and $\mathrm{Eu}(\mathrm{TTA})_{3}$ also indicate similar energy transfer results (Supporting Information, Fig. S4). We believe the energy transfer process in the Eu(TTA) ${ }_{3} @ B N$ system can be described as follows (Fig. $\left.5 \mathrm{c}\right)^{6}$ : (1) the $\mathrm{BN}$ host is excited from the ground state to the excited state by absorbing UV energy; (2) the energy of the BN 

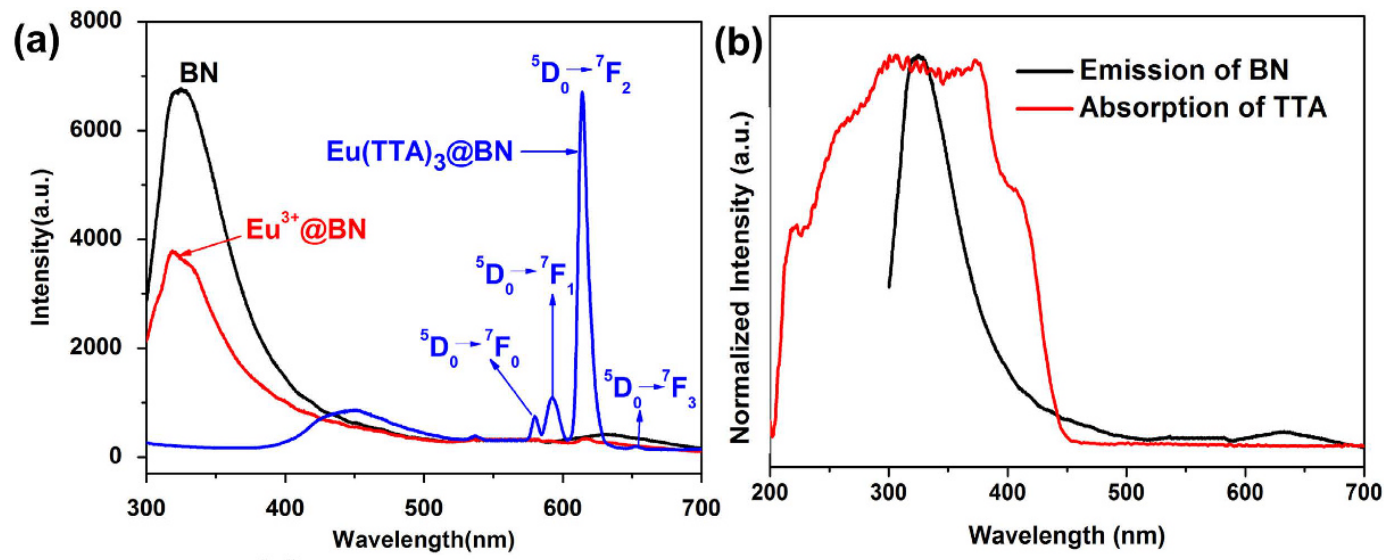

(c)

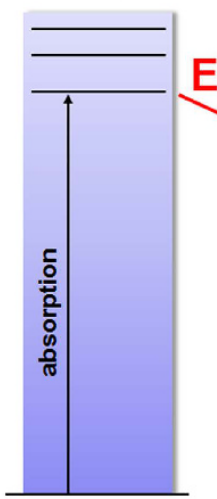

BN

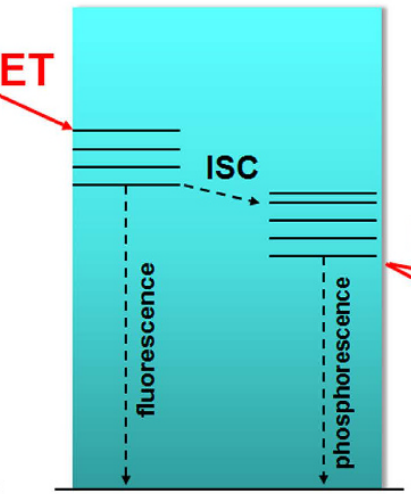

Ligands

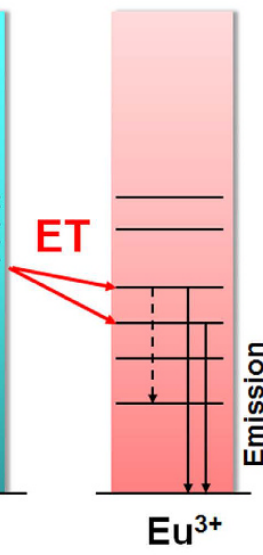

Figure 5. (a) Emission spectra of pure BN (black), Eu ${ }^{3+} @ B N\left(\right.$ red) and Eu(TTA) ${ }_{3} @ B N($ blue) samples. (b) Emission spectrum of pure BN (black) and UV-vis absorption spectrum of ligands TTA (red). (c) The schematic of the energy transfer process in $\mathrm{Eu}(\mathrm{TTA})_{3} @ \mathrm{BN}$ system. ET = energy transfer, ISC = intersystem crossing.

excited state is transferred to the ligands TTA; (3) the ligands TTA is excited to the singlet excited state, and then the energy is transferred to the triplet excited state through intersystem crossing (ISC); (4) energy transfers from the triplet state of the ligands to the excited $4 f$ states of $\mathrm{Eu}^{3+}$ ions; (5) red emission can be obtained through the $f-f$ transitions in $\mathrm{Eu}^{3+}$ ions. This interesting energy transfer process is quite different from those in earlier reported hybrid system, i.e. lanthanide complexes encapsulated in zeolites, SBA-15, MCM-41, etc, in which only effective energy transfer from the organic ligands to the $\mathrm{Ln}^{3+}$ ion (the so-called "antenna effects") takes place. Herein our

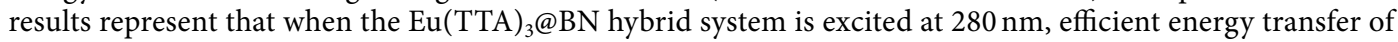
$\mathrm{BN}$-to-TTA-to-Eu ${ }^{3+}$ occurs, leading to the great enhancement of red emission.

We have also studied the PL properties of Eu(TTA) @BN samples with different Eu concentrations, as shown in Fig. S5, Supporting Information. All of the emission spectra consist of similar weak broad blue band emission and intense red emission peaks. With an increase of Eu contents from $0.00005 \mathrm{~mol} / \mathrm{L}$ to $0.005 \mathrm{~mol} / \mathrm{L}$, the intensity of blue band decreases gradually, while the intensity of red emission peaks increases significantly. However, when the Eu contents increases to $0.05 \mathrm{~mol} / \mathrm{L}$, a great decrease of red emission has been observed. The PL quenching may result from the nonradiative energy transfer process between $\mathrm{Eu}(\mathrm{TTA})_{3}$ in a high concentration.

The thermal stability of Eu(TTA) $)_{3}$ BN sample was studied by thermogravimetric (TG) analysis in the presence of air. Besides, the thermal stability of pure $\mathrm{Eu}(\mathrm{TTA})_{3} \cdot \mathrm{nH}_{2} \mathrm{O}$ was also studied for comparison. As shown in Fig. 6a, the curve of pure $\mathrm{Eu}(\mathrm{TTA})_{3} \cdot \mathrm{nH}_{2} \mathrm{O}$ complex shows a weight loss of $\sim 54 \%$ for the entire process. The first weight loss peak at $195^{\circ} \mathrm{C}$ corresponds to the loss of coordinate water, indicating the decomposition of the complexes starts. The weight loss occurs between $230^{\circ} \mathrm{C}$ and $550^{\circ} \mathrm{C}$ can be attribute to the decomposition of Eu(TTA) ${ }_{3}$. In detail, the second peak at $293^{\circ} \mathrm{C}$ is related to the decomposition of the three ligands in the complex, while the third peak appears at $507^{\circ} \mathrm{C}$ is due to the oxidation of the complex and finally $\mathrm{Eu}_{2} \mathrm{O}_{3}$ could be generated ${ }^{35}$. Figure 6b shows the TG curve of Eu(TTA $)_{3} @ B N$, displaying a weight loss of only $\sim 7.8 \%$ for the entire process. The first fast weight loss step starts from room temperature and ends at about $50{ }^{\circ} \mathrm{C}$, with a weight loss of $\sim 2 \%$, is related to the remaining gas desorption from the sample. The second step shows a weight loss peak at $355^{\circ} \mathrm{C}$ due to the decomposition of Eu(TTA $)_{3}$. The comparison indicates that the Eu(TTA $)_{3} @ B N$ hybrid material displays greatly enhanced thermal stability compared to the pure $\mathrm{Eu}(\mathrm{TTA})_{3} \cdot \mathrm{nH}_{2} \mathrm{O}$ complex. We believe that the space restriction of the europium (III) complexes by the pores of BN microfibers is the main factor for the improvement of their thermal stability. 

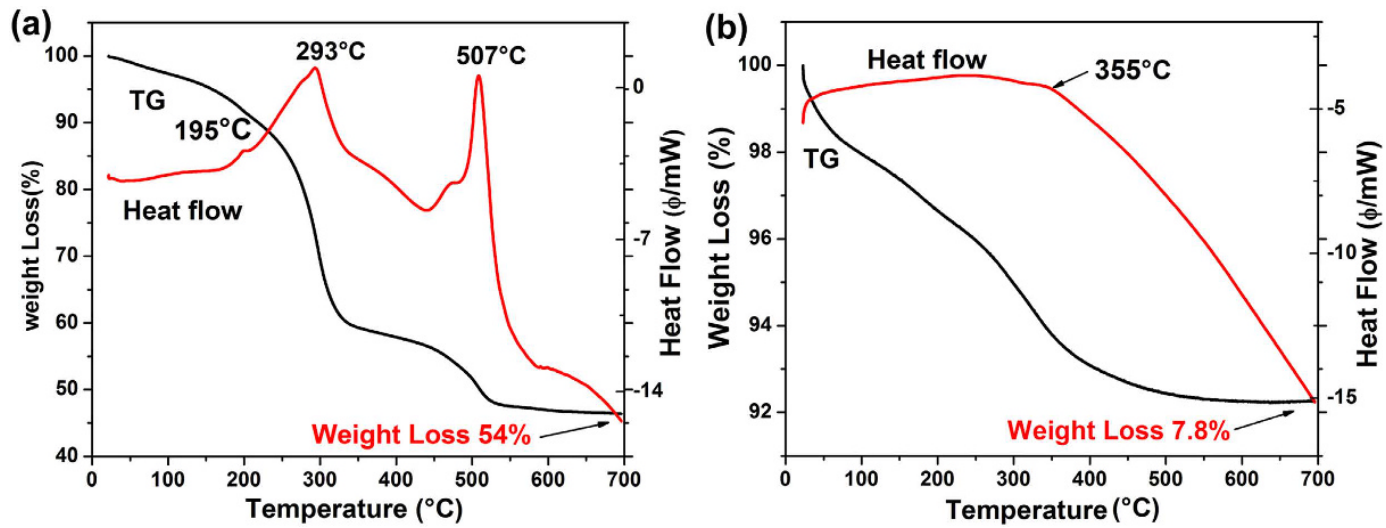

Figure 6. TG-DTA curves of (a) $\mathrm{Eu}(\mathrm{TTA})_{3} \cdot \mathrm{nH}_{2} \mathrm{O}$ and (b) $\mathrm{Eu}(\mathrm{TTA})_{3} @ \mathrm{BN}$, in the presence of air.

\section{Conclusions}

We designed and synthesized a novel kind of organic-inorganic hybrid materials via the incorporation of europium (III) complexes into 1D porous BN microfibers. TEM analysis demonstrated the amorphous europium (III) complexes were encapsulated inside porous BN, forming 1D Eu(TTA) $@$ BN hybrid composites. The developed Eu(TTA) $)_{3} @ B N$ hybrid composites showed bright visible red-light emission on UV illumination. Our results demonstrated an interesting and efficient energy transfer from BN host to TTA ligand and TTA ligand to Eu ${ }^{3+}$ ions occurred during the photoluminescence process, which was quite different from earlier reports regarding lanthanide complexes encapsulated within other porous matrix. The confinement of the europium (III) complexes within the pores of BN microfibers not only improved their thermal stabilities, but also decreased the aggregation-caused quenching in solid europium (III) complexes, leading to the remarkable increase of red emission. The designed synthetic approach we report here offers a great flexibility in rational design of many other hybrid luminescent materials based on porous BN.

\section{Experimental Section}

Staring materials. Porous $\mathrm{BN}$ microfibers were synthesized in relation to the reported procedure using $\mathrm{H}_{3} \mathrm{BO}_{3}$ and $\mathrm{C}_{3} \mathrm{~N}_{6} \mathrm{H}_{6}$ as the starting materials ${ }^{23}$. 2-Thenoyltrifluroacetone (99\%, TTA, Aldrich) was used as standard. $\mathrm{Eu}(\mathrm{TTA})_{3} \cdot \mathrm{nH}_{2} \mathrm{O}$ was produced by the reported method ${ }^{35}$. All the other reagents are analytical pure.

Preparation of Eu $\mathbf{E}^{3+} @ B N$ microfibers. Firstly, loading of $\mathrm{Eu}^{3+}$ ions into porous BN microfibers was achieved by adsorption method. Briefly, $1 \mathrm{~g}$ of porous $\mathrm{BN}$ microfibers were dispersed in $1 \mathrm{~L}$ of ethanol to obtain a $\mathrm{BN}$ suspension. Then $\mathrm{Eu}\left(\mathrm{NO}_{3}\right)_{3} \cdot 6 \mathrm{H}_{2} \mathrm{O}$ was dissolved in ethanol with a desired concentration $(0.0005 \mathrm{~mol} / \mathrm{L})$, and added dropwise into the $\mathrm{BN}$ suspension. Then the mixed solution was stirred for $12 \mathrm{~h}$ and filtered. After drying at $80^{\circ} \mathrm{C}$ in air overnight, white powder was obtained.

Synthesis of Eu(TTA) $)_{3} @ B N$ microfibers. Eu(TTA) $)_{3}$ BN microfibers were prepared by a gas diffusion method. In detail, $0.42 \mathrm{~g}$ of the as-prepared Eu ${ }^{3+} @ \mathrm{BN}$ white powder and $0.33 \mathrm{~g}$ of TTA were grinded together and then put into a gas diffusion flack. The flack was set inside of oil bath pan at $60^{\circ} \mathrm{C}$ for $1 \mathrm{~h}$ under a vacuum condition. Then the temperature was raised up to $100^{\circ} \mathrm{C}$ and kept for $24 \mathrm{~h}$. Finally, the products were washed with acetonitrile and dried in air at $80^{\circ} \mathrm{C}$, respectively.

Characterization. The structures of the as-prepared samples were characterized using an X-ray powder diffraction (XRD, BRUKER D8 FOCUS). The chemical bonding state of the products and the surface functional groups was examined by a Fourier transform infrared spectrophotometer (FTIR) (VECTOR22). The microstructures and compositions of the products were analysed using a transmission electron microscope (TEM, Philips Tecnai F20) equipped with an energy-dispersive X-ray spectrometer (EDS). For this, the powder samples were dispersed in ethanol, and then dripped dropwise onto the copper meshes covered with holey carbon films. The luminescent properties of the products were measured on the fluorescence spectrophotometer (F-7000 and FL3-22) and thermal stability analysis was carried out using a thermogravimetric analyzer (TG) (SDT Q-600) under normal atmosphere with stream of $\left(100 \mathrm{~mL} \mathrm{~min}^{-1}\right)$, at a heating rate of $5^{\circ} \mathrm{C} \mathrm{min}^{-1}$.

\section{References}

1. Dong, M. J., Zhao, M., Ou, S., Zou, C. \& Wu, C. D. A luminescent dye @MOF platform: emission fingerprint relationships of volatile organic molecules. Angew. Chem. Int. Ed. 53, 1575-1579 (2014).

2. Cui, Y. J. et al. Dye encapsulated metal-organic framework for warm-white LED with high color-rendering index. Adv. Funct. Mater. 25, 4796-4802 (2015).

3. Suresh, V. M., George, S. J. \& Maji, T. K. MOF nano-vesicles and toroids: self-assembled porous soft-hybrids for light harvesting. Adv. Funct. Mater. 23, 5585-5590 (2013).

4. Zhang, J. F. et al. Solid polymer electrolyte membranes based on organic/inorganic nanocomposites with star-shaped structure for high performance lithium ion battery. J. Membrane Sci. 509, 138-148 (2016).

5. Gao, Q. S., Chen, P., Zhang, Y. H. \& Tang, Y. Synthesis and characterization of organic-inorganic hybrid GeOx/Ethylenediamine nanowires. Adv. Mater. 20, 1837-1842 (2008). 
6. Feng, J. \& Zhang, H. J. Hybrid materials based on lanthanide organic complexes: a review. Chem. Soc. Rev. 42, 387-410 (2013).

7. Yan, B. Recent progress in photofunctional lanthanide hybrid materials. RSC Advances. 2, 9304-9324 (2012).

8. Binnemans, K. Lanthanide-Based Luminescent Hybrid Materials. Chem. Rev. 109, 4283-4374 (2009).

9. Li, P., Wang, Y. G., Li, H. R. \& Calzaferri, G. Luminescence enhancement after adding stoppers to europium (III) nanozeolite L. Angew. Chem. Int. Ed. 53, 2904-2909 (2014).

10. Li, H. R., Li, M., Wang, Y. \& Zhang, W. J. Luminescent hybrid materials based on laponite clay. Chem. Eur. J. 20, 10392-10396 (2014).

11. Wang, T. R., Li, P. \& Li, H. R. Color-tunable luminescence of organoclay-based hybrid materials showing potential applications in white LED and thermosensors. ACS Appl. Mater. Interfaces. 6, 12915-12921 (2014).

12. Yan, B. \& Zhou, B. Two photoactive lanthanide $\left(\mathrm{Eu}^{3+}, \mathrm{Tb}^{3+}\right)$ hybrid materials of modified $\beta$-diketone bridge directly covalently bonded mesoporous host (MCM-41). J. Photochem. Photobiol. A. 195, 314-322 (2008).

13. Li, Y. \& Yan, B. Lanthanide $\left(\mathrm{Tb}^{3+}, \mathrm{Eu}^{3+}\right)$ functionalized MCM-41 through modified Meta-aminobenzoic acid linkage: covalently bonding assembly, physical characterization and photoluminescence. Micropor. Mesopor. Mater. 128, 62-70 (2010).

14. Guo, X. M. et al. Incorporation of luminescent lanthanide complex inside the channels of organically modified mesoporous silica via template-ion exchange method. New J. Chem. 29, 1351-1358 (2005).

15. Peng, C. Y. et al. Synthesis, characterization, and luminescence properties of the ternary europium complex covalently bonded to mesoporous SBA-15, J. Phys. Chem. B 109, 15278-15287 (2005).

16. Li, S. W. et al. Improved photoluminescence properties of ternary terbium complexes in mesoporous molecule sieves. J. Phys. Chem. B 110, 23164-23169 (2006).

17. Kong, L. L., Yan, B. \& Li, Y. Hybrid materials of SBA-15 functionalized by $\mathrm{Tb}^{3+}$ complexes of modified acetylacetone: covalently bonded assembly and photoluminescence. J. Alloy. Comp. 481, 549-554 (2009).

18. Aisyiyah Jenie, S. N. et al. Lanthanide luminescence enhancements in porous silicon resonant microcavities. ACS Appl. Mater. Interface. 6, 12012-12021 (2014).

19. Carlos, L. D., Ferreira, R. A. S., Bermudez, V. de Z., Julián-López, B. \& Escribano, P. Progress on lanthanide-based organic-inorganic hybrid phosphors. Chem. Soc. Rev. 40, 536-549 (2011).

20. Han, W. Q., Brutchey, R., Tilley, T. D. \& Zettl, A. Activated boron nitride derived from activated carbon. Nano Lett. 4, 173-176 (2004).

21. Vinu, A. et al. Synthesis of mesoporous BN and BCN exhibiting large surface areas via templating methods. Chem. Mater. 17, 5887-5890 (2005).

22. Schlienger, S. et al. Micro-, mesoporous boron nitride-based materials templated from zeolites. Chem. Mater. 24, 88-96 (2012).

23. Li, J. et al. Porous boron nitride with a high surface area: hydrogen storage and water treatment. Nanotechnology. 24, 155603 (2013).

24. Li, J. et al. Activated boron nitride as an effective adsorbent for metal ions and organic pollutants. Sci. Rep. 3, 3208 (2013).

25. Weng, Q. H., Wang, X. B., Bando, Y. \& Golberg, D. One-step template-free synthesis of highly porous boron nitride microsponges for hydrogen storage. Adv. Energy Mater. 4, 1301525 (2014).

26. Weng, Q., Wang, X. B., Zhi, C. Y., Bando, Y. \& Golberg, D. Boron nitride porous microbelts for hydrogen storage. ACS Nano. 7, 1558-1565 (2013).

27. Lei, W. W., Portehault, D., Liu, D., Qin, S. \& Chen, Y. Porous boron nitride nanosheets for effective water cleaning. Nat. Commun. 4, 1777 (2013).

28. Lin, J. et al. Ultrafine porous boron nitride nanofibers synthesized via a freeze-drying and pyrolysis process and their adsorption properties. RSC Adv. 6, 1253-1259 (2016).

29. Zhi, C. Y. et al. Phonon characteristics and cathode luminescence of boron nitride nanotubes. Appl. Phys. Lett. 86, 213110 (2005).

30. Huang, Y. et al. Bulk synthesis, growth mechanism and properties of highly pure ultrafine boron nitride nanotubes with diameters of sub-10 nm. Nanotechnology. 22, 145602 (2011).

31. Sabbatini, N., Guardigli, M. \& Lehn, J. M. Luminescent lanthanide complexes as photochemical supramolecular devices. Coord. Chem. Rev. 123, 201-228 (1993).

32. Driesen, K., Van Deun. R., Görller-Walrand, C. \& Binnemans, K. Near-infrared luminescence of lanthanide calcein and lanthanide dipicolinate complexes doped into a silica-PEG hybrid material. Chem. Mater. 16, 1531-1535 (2004).

33. Zhang, X. H. et al. Blue emitting BCNO phosphors with high quantum yields. J. Mater. Chem. C 3, 3311-3317 (2015).

34. Zhang, X. H. et al. Effects of carbon and oxygen impurities on luminescence properties of BNCO phosphor. J. Am. Ceram. Soc. 97, 246 (2014).

35. Liu, Q. et al. Synthesis and luminescent properties of Eu(TTA) $)_{3} \cdot 3 \mathrm{H}_{2} \mathrm{O}$ nanocrystallines. Luminescence. 25, 307-310 (2010).

\section{Acknowledgements}

This work was supported by the National Natural Science Foundation of China (51372066, 51202055, 51402086, 51572068), the Program for Changjiang Scholars and Innovative Research Team in University (PCSIRT: IRT13060), the Hundred Talents Program of Hebei Province (E2014100011), the Natural Science Foundation of Hebei Province (E2016202122), and the Tianjin Research Program of Application Foundation and Advanced Technology (14JCYBJC42200).

\section{Author Contributions}

J.L. and Y.H. conceived and designed the experiments. J.L., C.F., X.H., W.W., Y.F. and J.L. performed the experiments and analyzed the data. J.L and Y.H. preformed the TEM characterization. J.L., C.F., Z.L., C.T. and Y.H. wrote the manuscript. All authors discussed and commented on the manuscript.

\section{Additional Information}

Supplementary information accompanies this paper at http://www.nature.com/srep

Competing financial interests: The authors declare no competing financial interests.

How to cite this article: Lin, J. et al. Europium (III) Organic Complexes in Porous Boron Nitride Microfibers: Efficient Hybrid Luminescent Material. Sci. Rep. 6, 34576; doi: 10.1038/srep34576 (2016).

This work is licensed under a Creative Commons Attribution 4.0 International License. The images or other third party material in this article are included in the article's Creative Commons license, unless indicated otherwise in the credit line; if the material is not included under the Creative Commons license, users will need to obtain permission from the license holder to reproduce the material. To view a copy of this license, visit http://creativecommons.org/licenses/by/4.0/

(C) The Author(s) 2016 